\title{
A whole genomic scan to detect selection signatures between Berkshire and Korean native pig breeds
}

Zewdu Edea and Kwan-Suk Kim

\begin{abstract}
Background: Scanning of the genome for selection signatures between breeds may play important role in understanding the underlie causes for observable phenotypic variations. The discovery of high density single nucleotide polymorphisms (SNPs) provide a useful starting point to perform genome-wide scan in pig populations in order to identify loci/candidate genes underlie phenotypic variation in pig breeds and facilitate genetic improvement programs. However, prior to this study genomic region under selection in commercially selected Berkshire and Korean native pig breeds has never been detected using high density SNP markers. To this end, we have genotyped 45 animals using Porcine SNP60 chip to detect selection signatures in the genome of the two breeds by using the $F_{S T}$ approach.

Results: In the comparison of Berkshire and KNP breeds using the FDIST approach, a total of 1108 outlier loci (3.48\%) were significantly different from zero at $99 \%$ confidence level with 870 of the outlier SNPs displaying high level of genetic differentiation $\left(F_{S T} \geq 0.490\right)$. The identified candidate genes were involved in a wide array of biological processes and molecular functions. Results revealed that 19 candidate genes were enriched in phosphate metabolism (GO: 0006796; ADCK1, ACYP1, CAMK2D, CDK13, CDK13, ERN1, GALK2, INPP1; MAK, MAP2K5, MAP3K1, MAPK14, P14KB, PIK3C3, PRKC1, PTPRK, RNASEL, THBS1, BRAF, VRK1). We have identified a set of candidate genes under selection and have known to be involved in growth, size and pork quality (CART, AGL, CF7L2, MAP2K5, DLK1, GLI3, CA3 and MC3R), ear morphology and size (HMGA2 and SOX5) stress response (ATF2, MSRB3, TMTC3 and SCAF8) and immune response ( HCST and RYR1).
\end{abstract}

Conclusions: Some of the genes may be used to facilitate genetic improvement programs. Our results also provide insights for better understanding of the process and influence of breed development on the pattern of genetic variations.

Keywords: Korean native pig, Genome-wide, SNP, Selection signature

\section{Background}

Pigs have long been of great economic importance to many farmers in the world. Molecular evidence supports independent domestication of pig in Asia and Europe from wild boar sub-species [1,2]. As compared to their wild ancestor, domestic pig breeds display a wide range of phenotypic variations that have been manipulated and shaped during the course of domestication and breed development for a wide range of traits. Some pig breeds,

\footnotetext{
* Correspondence: kwanskim@chungbuk.ac.kr

Department of Animal Science, Chungbuk National University, Cheongju
} 361-763, Korea

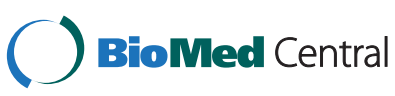

(c) 2014 Edea and Kim; licensee BioMed Central Ltd. This is an Open Access article distributed under the terms of the Creative Commons Attribution License (http://creativecommons.org/licenses/by/4.0), which permits unrestricted use, distribution, and reproduction in any medium, provided the original work is properly credited. The Creative Commons Public Domain Dedication waiver (http://creativecommons.org/publicdomain/zero/1.0/) applies to the data made available in this article unless otherwise stated. particularly commercial breeds have been intensively selection for better growth, meat quality and fertility traits may have resulted in loss of genetic diversity. To the contrast, most traditional breeds are reared by smallholder farmers and less subjected to selection pressure and harbor higher genetic diversity for adaptation under marginal environments.

The superiority of some commercial pig breeds for growth and carcass traits over traditional breeds, have led them to be the breed of choice and their continuous utilization in improvement of native populations through crossbreeding. In Asia, it has been known that commercial breeds have contributed to the genetic pool of most

rans 
indigenous breeds [3]. Likewise, for the last two decades, western pig breeds have been imported into the Korea peninsula and crossed with the Korean native pigs (KNP) in order to improve growth and carcass related traits [4]. As a result the number of Korean native pigs decreased noticeably following the introduction of improved breeds. Although commercial breeds are superior in terms of growth and feed efficiency traits, the Korean native pig harbors unique genetic material for product quality and better adaptation to low management levels [5]. Despite the indiscriminate crossbreeding, little is known regarding the genome composition difference between the Korean native and European (Berkshire) pig breeds.

There is a growing interest in spotting genomic regions or genes that have been under selection. $F_{S T}$ statistic is among the most widely used measures to identify genomic regions or loci that display high differentiation between populations [6]. Genomic regions or loci that show significantly high $F_{S T}$ values compared with neutral loci offer evidence for positive selection. Until recently, there has been little success of detecting genomic regions under selection in livestock species attributed to lack of high density molecular markers. However, through the advancement of high-throughput sequencing technology, thousands of single nucleotide polymorphisms have been discovered and open opportunities to facilitate and transform livestock genetic improvement programs. In pig, several thousands of SNPs spinning the whole genome has been discovered using next generation technologies [7]. The availability and discovery of such large number of SNPs provide a useful starting point to perform genomewide scan in pig populations in order to identify candidate genes underlining phenotypic variations between breeds. However, prior to this study genomic region under selection in commercially selected pig breed like Berkshire and Korean native pig has never been detected using high density SNP markers. Scanning of the genome for selection signature between highly selected and traditional breeds may play important role in identifying genes underlying for phenotypic variation. In addition, it can be used to facilitate genetic improvement and conservation programs. To this end, we have genotyped 45 animals are using Porcine SNP 60 BeadChip to identify loci variants showing directional selection in comparing European (Berkshire) and Korean native pig breeds using the $F_{S T}$ approach.

\section{Methods}

\section{Pig breeds, sampling and genotyping}

Samples were collected from unrelated Berkshire $(\mathrm{n}=29)$ and from Korea native pig $(K N P, n=16)$ breed. Briefly, Korean native pig was phenotypically discriminated as long black coarse hairs, long straight noses, greatly protruded mouth and straightly upright ears. The breed is known for its high prolificacy, better meat quality (high redness and intramuscular fat [5] and strong adaptability under low management conditions, but showed a slower growth rate, small adult body weight, smaller litter sizes, and lower carcass yield [8]. On the other hand, Berkshire pig breed is characterized by medium to large body size, fast growth rate, early maturing, and large litter size, medium and erect ears.

DNA samples of Korean native pig were obtained from National Institute of Animal Science (NIAS) and that of Berkshire were obtained from Dasan Breeding Farm in Korea. Sample collection procedures were approved by the National Institute of Animal Science (NIAS). During sample collection animals were treated humanely. All animals were genotyped performed using the Illumina Pocrine SNP60 BeadChip [9]. Common monomorphic SNPs for all of the breeds were discarded from further analyses. SNPs were filtered with criteria of call rate $(\geq 90 \%)$, minor allele frequency (MAF $\geq 5 \%$ ) and HardyWeinberg equilibrium (HWE $\geq 0.001$ ). Thus finally about 31,755 SNPs were considered for the study.

\section{Statistical analysis \\ Genetic variations}

Genetic diversity was assessed for each breed by calculating observed and expected heterozygosities using Arlequin software [10]. Principal component analysis was performed to illustrate the pattern of individual clustering using SNP and Variation Suite version 7 [11]. PCA assigns individuals to their population of origin using a common clustering algorithm Patterson et al. [12]. In the principal component analysis, the first principal component (PC1) accounts for the greater variation followed by principal component (PC2).

\section{Detection of outlier loci or signature of selection}

Detection of outlier loci was based on calculation of fixation index $\left(F_{S T}\right)$ at different significance levels as a measure of genetic differentiation for each locus between Berkshire and KNP following the FDIST approach proposed by [13] as implemented in Arlequin software [10]. Briefly, the FDIST program calculate genetic differentiation index $\left(F_{S T}\right)$ for each loci and then uses coalescent simulation to generate the null distribution of $F_{S T}$ values based on the infinite island model [13]. Within this framework, we ran 20,000 coalescent simulations to obtain the $\mathrm{P}$-values of locus-specific $F_{S T}$ conditioned on observed levels of heterozygosity with default settings. This method provides evidence for divergent selection by looking for outlier loci with FST values higher than expected, controlling for heterozygosity. The corresponding candidate genes for outlier SNPs $(\mathrm{P}<0.01)$ 
were annotated with the pig genome analysis data repository [14].

\section{Biological process and molecular functional analyses of the candidate genes under selection}

To known the biological process and molecular functional of each candidate genes, we assessed their Gene Ontology (GO) and classification using a web-based Database for Annotation, Visualization, and Integrated Discovery (DAVID) tools [15]. Furthermore, enrichment analysis was performed to identify biological processes and molecular functions over-represented by Fisher Exact test (EASE score). Any GO terms that have a larger than expected subset of selected genes were considered overrepresented and gave insight into the functional characteristics of the annotated genes.

\section{Haplotype blocks detection}

To investigate whether any of the significantly differentiated loci or genes $(\mathrm{P}<0.01)$ are in strong linkage disequilibrium, we further analyzed LD and haplotype blocks for the two breeds following the [16] method using the SNP and Variation Suite version 7 [11]. According to this method, SNP pairs to be in strong linkage disequilibrium (LD) if the one-sided upper 95\% confidence bound on D' is. 0.98 (that is, consistent with no historical recombination) and the lower bound is above 0.7.

\section{Results}

\section{Genetic diversity and population structure}

The average observed heterozygosity was $0.321 \pm 0.171$ in Berkshire and $0.326 \pm 0.173$ in Korean native pig, whereas the expected heterozygosity was found to be $0.319 \pm 0.156$ and $0.336 \pm 0.153$ for Berkshire and Korean native pig, respectively. The average within - breed fixation index $\left(F_{I S}\right)$ was shown deficiency of heterozygosity $(0.029)$ in Korean native pig whereas it was negative $(-0.008)$ in Berkshire. To illustrate the pattern of individual animals clustering, we performed principal component analyses (PCA). Principal component one (PC1) and principal component 2 (PC2) accounted for $82.33 \%$ and $17.67 \%$ of the total variance, respectively (Figure 1) and clearly separated individuals according to their breed group.

\section{Genetic differentiation, outlier loci and candidate genes under selection}

In this study, we are primarily intended to identify outlier loci in the comparison of two pig breeds (Berkshire and Korean native pigs). Level of differentiations between the breeds was measured by fixation indices. The overall $F_{S T}$ was 0.157 with about 29\% (9127) of the loci having an $F_{S T}$ value below zero or equal to zero. The highest genetic differentiation between the two breeds

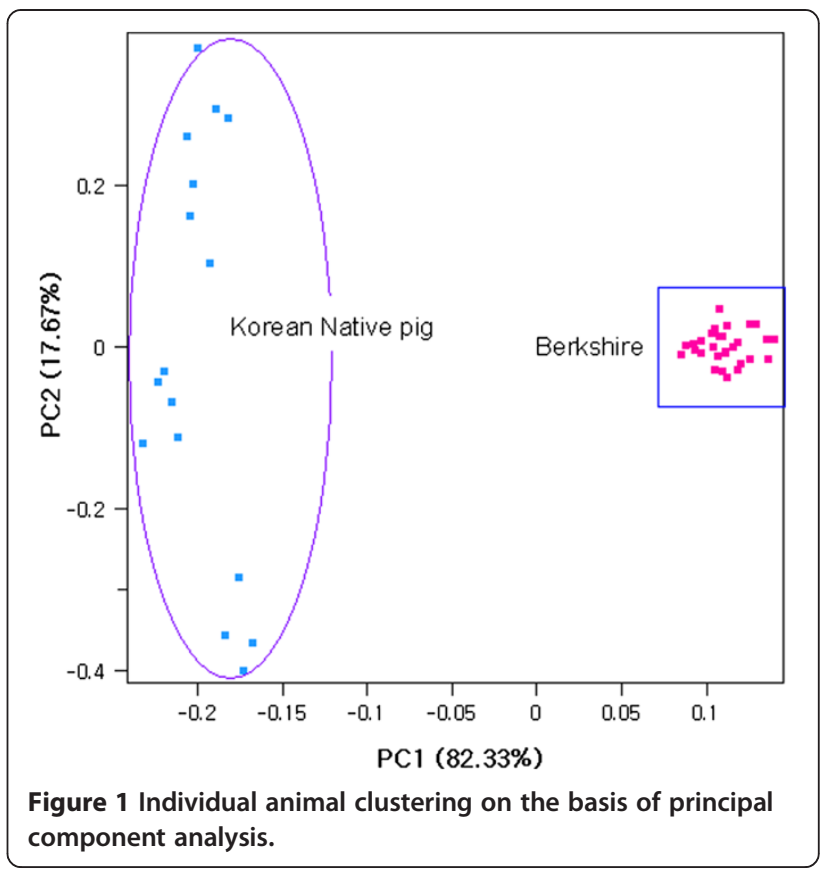

was observed on chromosome 16 where 7 SNPs or loci (rs81228734, rs81458940, rs81459172, rs81459185, rs 81459183 , rs81297918 and rs81459195) displayed an $F_{S T}$ value of 1 . In the comparison of Berkshire and KNP breeds, using the FDIST approach indicated that a total of 1108 loci $(3.48 \%)$ were significantly different from zero at $99 \%$ confidence level with 870 of the outlier SNPs displaying a high level of genetic differentiation $\left(\begin{array}{ll}F_{S T} & \geq 0.48\end{array}\right)$ (see Additional file 1) and revealing that the loci are potentially under directional selection. The distribution of $F_{S T}$ as a function of expected heterozygosity based on the 31755 loci is presented in Figure 2. The $F_{S T}$ value plot by chromosome is given in Additional file 2 .

\section{Haplotype blocks}

The distribution of haplotype blocks in Korean native pig and Berkshire are shown in Additional file 1: Table S1. The overall the distribution of haplotype blocks was higher in Korean native pig where a total of 76 variable size blocks was identified. In the contrast, in the Berkshire only 32 haplotype blocks were detected. The number of haplotype blocks detected in the Korean native pig population ranged from 10 in chromosome 5 to none in chromosome 6 and 9. In contrast, the Berkshire population had the highest number of haplotype blocks (7) in chromosome 7 and no haplotype block was identified for pairs of SNPs in chromosome 10,11, 14, 17 and 18. The size of each block varied from $5.716 \mathrm{~kb}$ to $158.824 \mathrm{~kb}$ in KNP and ranged from $11.916 \mathrm{~kb}$ to $158.270 \mathrm{~kb}$ in Berkshire samples. 


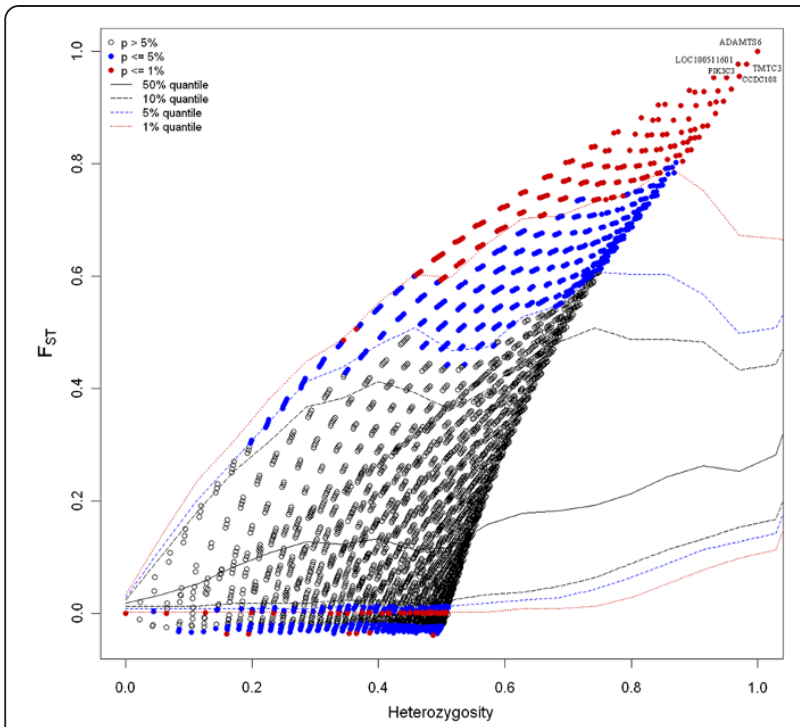

Figure 2 Joint distribution of $F_{S T}$ and heterozygosity based on the 31755 SNPs analyzed for Berkshire and Korean native pig breed comparison. Loci significant at 5\% and 1\% levels are indicated by blue and red circles, respectively, as estimated using FDIST approach of [12]. The red, blue, solid and broken lines represent the $1 \%, 5 \%, 10 \%$ and $50 \%$ quintiles, respectively, indicating the point at which $99 \%, 95 \%$ and $50 \%$ of the data fall above that value, respectively.

The first block, in chromosome 1 in Korean native pig covered $116.539 \mathrm{~kb}$ and contained IGF2R gene whereas the second block of the same chromosome spanned about $55.54 \mathrm{~kb}$ and covers SCAF8 gene. Two blocks in chromosome 5 (first and second blocks) which spanned $51.785 \mathrm{~kb}$ and $137.242 \mathrm{~kb}$, respectively encompassed WIFI candidate gene which is known to be associated with bone development. Similarly, the first block in chromosome 5 spanned about $51.785 \mathrm{~kb}$ and encompassed important gene (MSRB3) which is known to be related to cold and heat stresses. In chromosome 17, the third haplotype block covers MC3R gene previously known to be associated with body weight, adipose mass and feed conversion efficiency. The two breeds shared 12 common haplotye blocks. Of these blocks, block 69 in Korean native pig and block 28 in Berkshire spanned $158.27 \mathrm{~kb}$ and includes HOXA10 gene that play an important role in morphogenesis (Additional file 3). We compared the pattern of $F_{S T}$ and haplotype blocks distribution. The highest genetic differentiation $\left(F_{S T}=1.00\right)$ values were observed for SNPs positioned within chromosome 16 , but there was no any halotype block detected for these particular SNPs. Common for both breeds, the largest haplotype blocks were detected on chromosome 15 (87,700,078 - 87,163,500) with average $F_{S T}$ value of 0.82 , while the smallest haplotype $(12.848 \mathrm{~kb})$ was detected for chromosome 13 with these SNPs displaying an average genetic differentiation of 0.00 .
Gene ontology (GO) term analyses of the candidate genes under selection

The candidate genes were analyzed for their gene ontology (GO) and tested for enrichment based on a Fisher Exact test to identify biological process and molecular functions most pertinent by our genes list. The biological process and molecular functions of the candidate gene are present in Additional files 3 and 4, respectively. The identified candidate genes involved in a wide array of biological processes and molecular functions. Results revealed that 19 candidate genes were enriched in phosphate (GO: 0006796) and phosphorus metabolisms (GO: 0006793). GO term enriched in ion transport contained 16 candidate genes, while 5 (GLI3, CHD7, FBN2, HOXA10 and NR2F2) and 4 (GLI3, CHD7, FBN2 and HOXA10) candidate genes were involved in limb development (GO: 0060173) and morphogenesis (GO: 0035108), respectively. In addition, our functional analysis demonstrated that higher numbers of the candidate genes with significant enrichment had molecular functions related to ion binding (48). We also detected a significant encirclement for candidate genes involved in nucleotide and nucleoside bindings. Molecular function enriched with ATP bindings contained 23 candidate genes (ATP2A3, ADCK1, CAMK2D, CHD7, CDKL3, DGKB, ERN1, GALK2, KIF6, MAK, MAGI3, MAP2K5, MAP3K1, MAP3K14, MYO1B, PI4KB, PIK3C3, PRKCI, RNASEL, LOC392335, LOC441420, $M Y O 5 B, U B E 2 W, B R A F$ and VRK1) under selection. About 15 and 12 potential candidate genes were involved in protein kinase and protein serine/threonine kinase activity, respectively.

\section{Discussion}

The post-genomics era have opened opportunity in the scanning of whole for election signature in most commercial livestock species. Selection signatures may be used to identify genes or chromosomal regions that are possible targets of positive selection. The detection of selection signatures for local adaptation and phenotypic variations in Korean and western pig breeds is yet lacking. In this study, we compared two phenotypically distinct pig breeds in order to identify a sub-set loci significantly differentiated by employing an $F_{S T}$ test in porcine $60 \mathrm{~K}$ SNP chip. We detected 1108 outlier loci ( $p<0.01$ ) showing signature of selection and some of associated with genes known to be associated with production traits, ear size and morphology and diseases.

Growth and carcass qualities are important traits influencing the pig industry and these traits have been received considerable attention in breed improvement programs. Modern pigs have selected for lower levels of fat and fast lean growth [17]. In support with these facts, we identified potential candidate genes associated with growth, fat composition and feed conversion efficiency. Some of the genes 
include: CART, AGL, CF7L2, MAP2K5, DLK1 and MC3R. For example study by [18] revealed that chromosomal region harboring the CART gene is a promising QTL in pig production traits (abdominal fat, weight and back fat thickness). In addition, this gene plays a crucial role in a variety of physiological processes, including food intake and body weight regulation [19]. Recent genome-wide association studies (GWAS) found that genetic polymorphisms in the $A G L$ gene has shown to be association with growth and carcass traits in the crossbred population of Landrace and Jeju (Korea) Black pig [20]. We also detected signature of selection at TCF7L2 loci which found to be associated with fat deposition traits in pigs [17]. This gene is known to locate on chromosome 14 where chromosome - wide significant trait loci for last ribs back fat and carcass weight were detected in Berkshire and Yorkshire crosses population [21].

Another important candidate gene under selection is MAP2K5. MAP2K5 associated with body mass index and obesity in human [22]. Furthermore this gene is a component of the MAPK-family intracellular signaling pathways, responding to extracellular growth factors 2 (IGF2) [23]. Interestingly, we detected selection signatures at DLK1 which is one of among imprinted genes in the callipyge locus (CLPG) region and associated with fat deposition, lean muscle mass and prenatal and postnatal growth rates in pigs [24]. In the comparison of the small sized KNP against the medium sized Berkshire, we identified selection footprints in HMGA2 and CA3 genes which were previously known to be associated with meat quality traits [25].

We have additionally identified two candidate genes, GLI3 and MC3R, that known to influence body weight and growth traits. GLI3 is associated with growth traits [26]. Melanocortin-3 receptor (MC3R) was previously reported to affect adipose mass in mice [27]. This gene is also associated with feed conversion and body weight in broiler [28] and with body weight in cattle [29]. KNP grow slower compared to the faster growing ability of European commercial pig breed (Berkshire). These genes may be involved for observable phenotypic variations in terms of growth traits in the two breeds and could serve as a molecular marker in the breeding programs. Therefore, our study provides evidence that these candidate genes detected here are likely under selection for better carcass quality traits and may be used for marker-assisted selection in beef cattle breeding program.

Among the potential candidate genes displaying signature of selection signature are ATF2, MSRB3, TMTC3 and $S C A F 8$ genes which were involved in stress responses [30,31]. Particularly the MSRB3 gene is known to play a key role in protection mechanisms against cold and heat stresses [32]. Considering the extreme environmental temperature where KNP is originated and developed, this gene is likely under selection for cold resistance.

Breed difference for disease resistance quite obvious between improved and native breeds. KNP is known to have adaptability under low management systems. We identified genes known to playing physiological functions of either inhabiting or activating immune response. These include pig immune receptor (HCST or DAP10) which was detected predominantly in lymphohematopoietic tissues [33]. Studies in humans and mice demonstrated that DAP1O and DAP12 can either activate or inhibit immune responses [34] implying that they play an important role in innate immune responses. Malignant hyperthermia $(\mathrm{MH})$ causes major economic losses in the swine industry. Interestingly, here we detected signature of selection in $R Y R 1$ gene which is an essential gene in swine. A single point mutation in the RYRI gene was found to be correlated with $\mathrm{MH}$ in breeds of swine [35].

Pigs have undergone morphological evolution through the course of domestication and breed development. For instance, strong selection signatures have been detected in loci harboring quantitative trait loci that explain morphological changes in the domestic pig [36]. In line with this evidence, our GO classification analysis indicated that some of the candidate genes under selection were known to be associated with limb development and morphogenesis. Among others, ear size and morphology are important conformation characteristics for breed discrimination. In this study we detected to two potential candidate genes (HMGA2 and SOX5) known to have a key role in affecting ear size and morphology. HMGA2-LPP fusion protein promotes chondrogenesis [37]. More interestingly, studies revealed that HMGA2-deficient mice develop smaller ears [38] and in dogs, it may be involved in differences in the size and type of ears [39]. Furthermore, SOX5 plays a role in chondrogenesis [40]. Considering the distinct variations in ear morphology and size displayed by the study populations, the detected genes are potentially under selection for the observable differences. Considerable variation was observed regarding to the number and distribution of haplotype blocks in the two breeds. The relatively higher number of haplotype blocks detected in the KNP population is consistence with the demographic history of the breed [4]. The bottle neck associated with reduction in KNP population may lead to greater LD.

Modern pig breeds have been selected for reproduction traits. KNP is known for its high prolificacy and here we identified some potential genes having role in reproduction or fertility. Possible role of phospholipase B in sperm maturation and activation was investigated in guinea pig [41]. KIF6 or KRP3 gene has involved in spermatid maturation mediated by possible interaction with the Ran GTPase [42]. Previously PDE3A is identified as 
the major cAMP-degrading PDE in the oocyte and regulates the resumption of meiosis [43].

\section{Conclusions}

In this study we identified several candidate genes which have known associated with pork production (growth, size, and pork quality), morphology, stress and immune response. Some of the genes may be used to facilitate genetic improvement programs. Our results also provide insights for better understanding of the process and influence of breed development on the pattern of genetic variations. As the current annotation of pig genome is not conclusive, it is worth noting that many of the outlier loci or genes without GO terms may have relevant biological meanings and functions.

\section{Additional files}

Additional file 1: Loci displaying selection signature at $99 \%$ confidence level and candidate genes in the comparison of Berkshire and Korean native pig breeds.

Additional file 2: $F_{S T}$ plot by chromosome in the comparison of Koran native and Berkshire pig breeds.

Additional file 3: Biological process of the candidate genes under selection based on gene ontology (GO) analysis.

Additional file 4: Molecular functions of the candidate genes under selection based on gene ontology (GO) analysis.

\section{Abbreviations}

DAVID: Database for Annotation, Visualization, and Integrated Discovery; DNA: Deoxyribonucleic acid; EASE: Expression analysis systematic explorer; $F_{I S}$ : Within - breed fixation index; $F_{S T}$ : Fixation index; GO: Gene ontology; ILRI: International Livestock Research Institute; HWE: Hardy-Weinberg equilibrium; KNP: Korean native pig; MAF: Minor allele frequency; NIAS: National Institute of Animal Science; PC: Principal component: PCA: Principal component analysis; SNP: Single nucleotide polymorphism.

Competing interests

The authors declare that they have no competing interests.

\section{Authors' contributions}

ZE analyzed data and wrote this paper; KSK conceived this research and edited the paper. Both authors read and approved the final manuscript.

\section{Authors' information}

E.Z PhD student at Chungbuk National University and graduate fellow at ILRI; K.S.K professor of Animal Science at Chungbuk National University.

\section{Acknowledgements}

This work was supported by a grant entitled "Development of Genetic Improvement Systems in Pigs Using Genomic and Reproductive Technologies" from the Korea Institute of Planning and Evaluation for Technology of Food, Agriculture, Forestry and Fisheries and 2-7-10 Agenda Research (PJ00670701) from the National Institute of Animal Science.

Received: 29 June 2014 Accepted: 26 October 2014

Published: 6 November 2014

\section{References}

1. Giuffra E, Kijas JM, Amarger V, Carlborg O, Jeon JT, Andersson L: The origin of the domestic pig: Independent domestication and subsequent introgression. Genetics 2000, 154:1785-1791.

2. Larson G, Dobney K, Albarella U, Fang M, Matisoo-Smith E, Robins J, Lowden S, Finlayson $\mathrm{H}$, Brand T, Willerslew E, Rowley-Conwy P, Andersson L, Cooper A:
Worldwide phylogeography of wild boar reveals multiple centers of pig domestication. Science 2005, 307:1618-1621.

3. Yang SL, Wang ZG, Liu B, Zhang GX, Zhao SH, Yu M, Fan B, Li MH, Xiong TA, Li K: Genetic variation and relationships of eighteen Chinese indigenous pig breeds. Genet Sel Evol 2003, 35:657-671.

4. Kim TH, Kim KS, Choi BH, Yoon DH, Jang GW, Lee KT, Chung HY, Lee HY, Park HS, Lee JW: Genetic structure of pig breeds from Korea and China using microsatellite loci analysis. J Anim Sci 2005, 83:2255-2263.

5. Park BY, Kim NK, Lee CS, Hwang $I \mathrm{H}$ : Effect of fiber type on postmortem proteolysis in longisimus muscle of Landrace and Korean native black pigs. Meat Sci 2007, 77:482-491.

6. Porto-Neto LR, Lee SH, Lee HK, Gondro C: Detection of signatures of selection using Fst. Methods Mol Biol 2013, 1019:423-436.

7. Ramos AM, Crooijmans RP, Affara NA, Amaral AJ, Archibald AL, Beever JE, Bendixen C, Churcher C, Clark R, Dehais P, Hansen MS, Hedegaard J, Hu ZL, Kerstens HH, Law AS, Megens H-J, Milan D, Nonneman DJ, Rohrer GA, Rothschild MF, Smith TPL, Schnabel RD, Van Tassell CP, Taylor JF, Wiedmann RT, Schook LB, Groene MAM: Design of a high density SNP genotyping assay in the pig using SNPs identified and characterized by next generation sequencing technology. PLoS One 2009, 4:e6524.

8. Hwang $\mathrm{IH}$, Park BY, Cho SH, Kim JH, Lee JM: Identification of muscle proteins related to objective meat quality in Korean native black pig. Asian-Australas J Anim Sci 2004, 17:1599-1607.

9. Illumina. [http://www.llumina.com]

10. Excoffier $\mathrm{L}$, Lischer $\mathrm{H}$ : Arlequin: An integrated software package for population genetics data analysis. Evol Bioinform 2011, 1:47-50.

11. SNP and Variation Suite Version 7. [http://www.goldenhelix.com]

12. Patterson N, Price A, Reich D: Population Structure and Eigen analysis. PLoS Genet 2006, 2006(2):e190.

13. Beaumont MA, Nichols RA: Evaluating loci for use in the genetic analysis of population structure. Proc R SOC B 1996, 263:1619-1626.

14. ORG Data Repository. [http://www.animalgenome.org/repository/pig/]

15. Database for Annotation, Visualization, and Integrated Discovery (DAVID) tools. [http://david.abcc.ncifcrf.gov/]

16. Gabriel SB, Schaffner SF, Nguyen H, Moore JM, Roy J, Blumenstiel B, Higgins J, DeFelice M, Lochner A, Faggart M, Liu-Cordero SN, Rotimi C, Adeyemo A, Cooper R, Ward R, Lander ES, Daly MJ, Altshuler D: The Structure of Haplotype Blocks in the Human Genome. Science 2002, 296:2225. doi:10.1126/science.1069424.

17. Du ZQ, Fan B, Zhao X, Amoako R, Rothschild MF: Association analyses between type 2 diabetes genes and obesity traits in pigs. Obesity 2009, 17(17):323-329.

18. Stachowiak M, Cieslak J, Skorczyk A, Nowakowska J, Szczerbal I, Szydlowski M, Switonski M: The pig CART (cocaine- and amphetamine-regulated transcript) gene and association of its microsatellite polymorphism with production traits. J Anim Breed Genet 2009, 126:37-42.

19. Vicentic A, Jones DC: The CART system in appetite and drug addiction. J Pharmacol Exp Ther 2007, 320:499-506.

20. Han SH, Shin KY, Lee SS, Ko MS, Jeong DK, Oh HS, Yang BC, Cho IC: SINE indel polymorphism of AGL gene and association with growth and carcass traits in Landrace $\mathrm{x}$ Jeju Black pig F(2) population. Mol Biol Rep 2010, 37:467-471.

21. Malek M, Dekkers JC, Lee HK, Baas TJ, Prusa K, Huff-Lonergan E, Rothschild MF: A molecular genome scan analysis to identify chromosomal regions influencing economic traits in the pig. II. Meat and muscle composition. Mamm Genome 2001, 12:637-645.

22. Rask-Andersen M, Jacobsson JA, Moschonis G, Ek AE, Chrousos GP, Marcus C, Manios Y, Fredriksson R, Schiöth HB: The MAP2K5-linked SNP rs2241423 is associated with BMI and obesity in two cohorts of Swedish and Greek children. BMC Med Genet 2012, 13:36. doi:10.1186/ 1471-2350-13-36.

23. Carter EJ, Cosgrove RA, Gonzalez I, Eisemann JH, Lovett FA, Cobb LJ, Pell JM: MEK5 and ERK5 are mediators of the pro-myogenic actions of IGF-2. J Cell Sci 2009, 122:3104-3112.

24. Kim KS, Kim JJ, Dekkers JCM, Rothschild MF: Polar overdominant inheritance of a DLK1 polymorphism is associated with growth and fatness in pigs. Mamm Genome 2003, 15:555-559.

25. Wimmers K, Murani E, Te Pas MF, Chang KC, Davoli R, Merks JW, Henne H, Muraniova M, da Costa N, Harlizius B, Schellander K, Böll I, Braglia S, de Wit AA, Cagnazzo M, Fontanesi L, Prins D, Ponsuksili S: Associations of functional candidate genes derived from gene-expression profiles of 
prenatal porcine muscle tissue with meat quality and muscle deposition. Anim Genet 2007, 38:474-484.

26. Huang YZ, Wang KY, He H, Shen QW, Lei CZ, Lan XY, Zhang CL, Chen H: Haplotype distribution in the GLI3 gene and their associations with growth traits in cattle. Gene 2013, 513:141-146.

27. Butler AA, Kesterson RA, Khong K, Cullen MJ, Pelleymounter MA, Dekoning J, Baetscher M, Cone RD: A unique metabolic syndrome causes obesity in the melanocortin-3 receptor-deficient mouse. Endocrinology 2000, 141:3518-3521.

28. Sharma P, Bottje W, Okimoto R: Polymorphisms in uncoupling protein, melanocortin 3 receptor, melanocortin 4 receptor, and proopiomelanocortin genes and association with production traits in a commercial broiler line. Poult Sci 2008, 87:2073-2086.

29. Luoreng ZM, Wang XP, Ma Y, Li F, Guo DS, Li N, Wang JR: Three novel SNPs in the coding region of the Bovine MC3R gene and their aassociations with ggrowth traits. Biochem Genet 2014, 52:116-124.

30. Liu S, Wang F, Yan L, Zhang L, Song Y, Xi S, Jia J, Sun G: Oxidative stress and MAPK involved into ATF2 expression in immortalized human urothelial cells treated by arsenic. Arch Toxicol 2013, 87:981-989.

31. Racapé M, Duong Van Huyen JP, Danger R, Giral M, Bleicher F, Foucher Y, Pallier A, Pilet P, Tafelmeyer P, Ashton-Chess J, Dugast E, Pettré S, Charreau B, Soulillou JP, Brouard S: The involvement of SMILE/TMTC3 in endoplasmic reticulum stress response. PLoS One 2011, 6:e19321.

32. Lim DH, Han JY, Kim JR, Lee YS, Kim HY: Methionine sulfoxide reductase $B$ in the endoplasmic reticulum is critical for stress resistance and aging in Drosophila. Biochem Biophys Res Commun 2012, 419:20-269.

33. Yim D, Jie HB, Sotiriadis J, Kim YS, Kim KS, Rothschild MF, Lanier LL, Kim YB: Molecular cloning and characterization of pig immunoreceptor DAP10 and NKG2D. Immunogenetics 2001, 53:243-249.

34. Lanier LL: DAP10- and DAP12-associated receptors in innate immunity. Immunol Rev 2009, 227:150-160.

35. Fujji J, Otsu K, Zorzato F, Leon SD, Khanna VK, Weiler JE, O'Brien PJ, MacLannan DH: Identification of a mutation in porcine ryanodine receptor associated with malignant hyperthermia. Science 1991, 253:448-451.

36. Rubin C, Megens HJ, Barrio AM, Maqbool K, Sayyab S, Schwochow D, Wang C, Carlborg Ö, Jern P, Jørgensen CB, Archibald AL, Fredholm M, Groenen MA, Andersson L: Strong signatures of selection in the domestic pig genome. PNAS 2012, 2012(109):19529-1936.

37. Kubo T, Matsui Y, Goto T, Yukata K, Yasui N: Over expression of HMGA2- LPP fusion transcripts promotes expression of the alpha 2 type XI collagen gene. Biochem Biophys Res Commun 2006, 340:476-481.

38. Xiang X, Benson KF, Chada K: Mini-mouse: disruption of the pygmy locus in a transgenic insertional mutant. Science 1990, 247:967-969.

39. Boyko AR, Quignon P, Li L, Schoenebeck JJ, Degenhardt JD, Lohmueller KE, Zhao K, Brisbin A, Parker HG, von Holdt BM, Cargill M, Auton A, Reynolds A, Elkahloun AG, Castelhano M, Mosher DS, Sutter NB, Johnson GS, Novembre J, Hubisz MJ, Siepel A, Wayne RK, Bustamante CD, Ostrander EA: A simple genetic architecture underlies morphological variation in dogs. PLOS Biol 2010, 8:e1000451.

40. Smits P, Li P, Mandel J, Zhang Z, Deng JM, Behringer RR, de Crombrugghe B, Lefebvre V: The transcription factors L-Sox5 and Sox6 are essential for cartilage formation. Dev Cell 2001, 1:277-290.

41. Delagebeaudeuf C, Gassama-Diagne A, Nauze M, Ragab A, Li RY, Capdevielle J, Ferrara P, Fauvel J, Chap H: Ectopic epididymal expression of guinea pig intestinal phospholipase B. Possible role in sperm maturation and activation by limited proteolytic digestion. J Biol Chem 1998, 273:13407-13414

42. Zou Y, Millette CF, Sperry AO, KRP3A and KRP3B: Candidate motors in spermatid maturation in the seminiferous epithelium. Biol Reprod 2002, 66:843-855.

43. Sasseville M, Côté N, Guillemette C, Richard FJ: New insight into the role of phosphodiesterase 3A in porcine oocyte maturation. BMC Dev Biol 2006, 6:47.

\section{doi:10.1186/2055-0391-56-23}

Cite this article as: Edea and Kim: A whole genomic scan to detect selection signatures between Berkshire and Korean native pig breeds. Journal of Animal Science and Technology 2014 56:23.

\section{Submit your next manuscript to BioMed Central and take full advantage of:}

- Convenient online submission

- Thorough peer review

- No space constraints or color figure charges

- Immediate publication on acceptance

- Inclusion in PubMed, CAS, Scopus and Google Scholar

- Research which is freely available for redistribution 